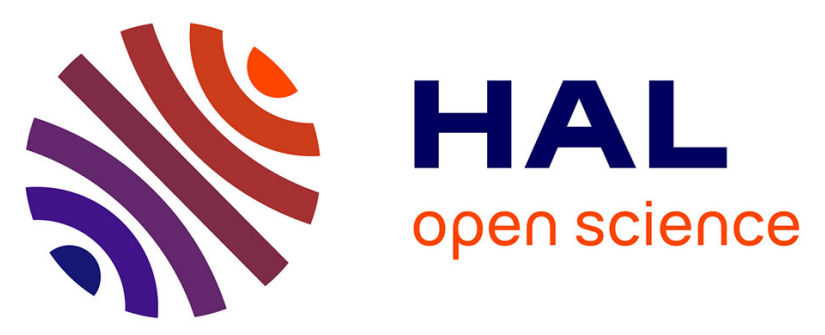

\title{
Measuring social inequalities in cause-specific mortality in France: comparison between linked and unlinked approaches.
}

Grégoire Rey, Stéphane Rican, Danièle Luce, Gwenaelle Menvielle, Eric Jougla

\section{To cite this version:}

Grégoire Rey, Stéphane Rican, Danièle Luce, Gwenaelle Menvielle, Eric Jougla. Measuring social inequalities in cause-specific mortality in France: comparison between linked and unlinked approaches.. Epidemiology and Public Health = Revue d'Epidémiologie et de Santé Publique, 2013, 61 (3), pp.22131. 10.1016/j.respe.2012.11.004 . inserm-00844594

\section{HAL Id: inserm-00844594 https://www.hal.inserm.fr/inserm-00844594}

Submitted on 15 Jul 2013

HAL is a multi-disciplinary open access archive for the deposit and dissemination of scientific research documents, whether they are published or not. The documents may come from teaching and research institutions in France or abroad, or from public or private research centers.
L'archive ouverte pluridisciplinaire HAL, est destinée au dépôt et à la diffusion de documents scientifiques de niveau recherche, publiés ou non, émanant des établissements d'enseignement et de recherche français ou étrangers, des laboratoires publics ou privés. 


\section{La mesure des inégalités sociales de mortalité par cause en France : Comparaison des approches chaînée et non chaînée}

Measuring social inequalities in cause-specific mortality in France: Comparison between linked and unlinked approaches

G. Rey ${ }^{1}$, S. Rican ${ }^{1,2}$, D. Luce $^{3,4}$, G. Menvielle ${ }^{3,4}$, E. Jougla ${ }^{1}$

${ }^{1}$ Inserm, CépiDc, Le Kremlin-Bicêtre, France

${ }^{2}$ Laboratoire Espace Santé et Territoires, Nanterre, France

${ }^{3}$ Inserm U1018, CESP Centre de recherche en Épidémiologie et Santé des Populations, UMRS 1018, Epidémiologie des déterminants professionnels et sociaux de la santé, Villejuif, France

${ }^{4}$ UVSQ: University of Versailles St-Quentin, UMRS 1018, Villejuif, France

Auteur correspondant :

Grégoire Rey

Inserm, CépiDc

80 rue du Général Leclerc

94270 Le Kremlin-Bicêtre

Tel. : 0149591863

Fax : 0149591930

gregoire.rey@inserm.fr

Titre courant :

Comparaison des approches chaînée et non chaînée 


\section{Abstract}

\section{Background}

Monitoring the time trends in socio-economic inequalities in mortality by cause is a key public health issue. The aim of this study is to present the state of available data to measure social inequalities in cause-specific mortality in the French population aged 25 to 55 . More specifically, it compares bias and precision related to the use of occupational class declared at the last census (linked data) to the one declared at the mortality civil state (unlinked data).

\section{Methods}

We used a representative sample of $1 \%$ of the French population. Causes of death were obtained by direct linkage with the French national death registry. Occupational class was classified into eight categories.

Taking professionals and managers as the reference, relative risks of mortality by cause and their 95\% confidence intervals were estimated using Poisson models for the 1983-1989, 1991-1997 and 2000-2006 periods. The relative risks were calculated both with linked data and exhaustive unlinked data.

\section{Results}

Over the 2000-2006 period, occupational class declared at census and at civil state are consistent for half of the deaths. Relative risks for manual workers are similar between the two approaches over the 1983-1989 and 1991-1997 periods, and higher for the unlinked approach over the 2000-2006 period. Over the latter period, the order and magnitude of relative risks are varying similarly by occupational class and cause of death for both approaches. Confidence intervals obtained from linked data are large.

\section{Conclusion}

Occupational class derived from the mortality civil state has to be exploited with cautious as a measure for epidemiological purpose and available linked data do not allow accurate estimates of social inequalities in cause-specific mortality. Other solutions should be considered in order to improve the follow-up of mortality social inequalities in mortality. Among them: the collection of education level at the mortality civil state or the linkage of the cause of death database with other exhaustive and informative databases.

Keywords: Mortality. Inequalities. Occupational class. Measures. Causes of death 


\section{Résumé}

\section{Position du problème}

Le suivi dans le temps des inégalités sociales de mortalité représente un enjeu de santé publique majeur. Cette étude propose de présenter les données disponibles permettant d'évaluer les différentiels socio-économiques de mortalité par cause dans la population âgée de 25 à 55 ans en France. Plus spécifiquement, elle compare le biais et la précision inhérents à l'utilisation de la profession et catégorie socio-professionnelle (PCS) déclarée lors du dernier recensement (méthode chaînée dans l'échantillon démographique permanent (EDP)) ou à l'état civil au moment du décès (approche non chaînée exhaustive).

\section{Méthodes}

L'EDP, un échantillon au centième de la population vivant en France, apparié aux causes médicales de décès a été utilisé pour l'approche chaînée. Les données exhaustives de mortalité par cause ont été utilisées pour l'approche non chaînée.

Les PCS ont été classées en huit catégories.

En prenant les cadres comme référence, des risques relatifs de mortalité par cause et leur intervalle de confiance à 95\% ont été calculés à l'aide de régression de Poisson pour les périodes 1983-1989, 1991-1997 et 2000-2006. Ces calculs ont été faits sur la base de l'EDP et sur les données exhaustives non chaînées.

\section{Résultats}

Sur la période 2000-2006, les PCS déclarées au précédent recensement et à l'état civil concordent pour la moitié des décès de l'EDP. Lorsque l'on compare les deux approches, les risques relatifs sont généralement plus élevés avec l'approche non chaînée, en particulier pour les employés. Les risques relatifs pour les ouvriers sont similaires selon les deux approches sur les périodes 1983-1989 et 1991-1997 et plus élevés pour l'approche non chaînée sur la période 2000-2006. Sur cette dernière période, l'ordre et l'amplitude des risques relatifs varient de façon similaire selon la PCS et la cause de décès pour les deux approches. Les intervalles de confiance obtenus à l'aide de l'approche chaînée sont larges.

\section{Conclusion}

La PCS déclarée à l'état civil au moment du décès nécessite des précautions importantes pour un usage en épidémiologie et les données de l'EDP ne permettent pas un suivi fin des inégalités sociales de mortalité. D'autres solutions sont envisageables comme le recueil du niveau d'étude à l'état civil ou l'appariement de la base de mortalité par cause de décès avec d'autres bases de données à vocation exhaustive.

Mots-clés : Mortalité. Inégalités. Statut Socio-économique. Mesure. Causes de décès 3 


\section{Introduction}

La réduction des inégalités sociales de santé constitue aujourd'hui un des objectifs majeurs des politiques de santé publique en France (1). Pour évaluer cet objectif, il faut disposer d'outils de mesure fiables permettant un suivi dans le temps de ces inégalités. De nombreuses pistes sont aujourd'hui avancées pour approfondir ces outils de mesure en France $(2,3)$.

La mesure des inégalités sociales passe par le choix d'indicateurs de santé et d'indicateurs liés au statut socioéconomique. Ces derniers sont le plus souvent envisagés sous l'angle des catégories socio-professionnelles (PCS), du niveau d'études ou du niveau de revenu (4). Concernant le choix des indicateurs sanitaires, la mortalité par cause de décès, bien que ne reflétant qu'un versant négatif de l'état de santé d'une population au détriment d'autres dimensions sanitaires telle que la qualité de vie, constitue à ce jour l'une des mesures les plus aisément mobilisables. L'exhaustivité de l'information et l'effort international important visant à l'homogénéisation de son recueil permet de la considérer comme une source fiable pour effectuer des comparaisons dans le temps et dans l'espace, sur différentes pathologies. Elle est régulièrement utilisée, en particulier depuis la loi de santé publique de 2004 comme une information sanitaire de référence pour le pilotage des politiques de santé publique (5).

Pour mesurer les inégalités sociales de mortalité par cause, deux types de données sont utilisés : des données longitudinales, dites "chaînées", permettent la mesure des différentiels de mortalité selon les catégories sociales collectées en début de suivi, et des données, dites "non chaînées", rapportent le nombre de décès selon la catégorie sociale déclarée à l'état civil au moment du décès aux effectifs estimés de population par catégorie sociale lors des recensements. 
Les mesures de différentiels de mortalité par catégorie sociale obtenues à partir de données non chaînées sont généralement les plus aisées à produire. Historiquement, elles ont permis les premières mesures de différentiels sociaux de mortalité en Grande-Bretagne (6) et en France (7-9).

Plusieurs limites sont rapportées pour cette approche : elle porte d'une part sur la noncomparabilité des catégories sociales déclarées à l'état civil au moment du décès et de celles déclarées par le sujet de son vivant, responsable de discordances entre numérateur et dénominateur (10-12). Les personnes déclarant la catégorie sociale sont différentes (les proches au moment du décès vs. le sujet lui-même) et les questions ne sont pas formulées de façon identiques dans le bulletin d'Etat civil (une ligne "profession") et le recensement (plusieurs questions détaillées) (13). Ce biais "numérateur-dénominateur" est potentiellement variable selon les pays : relativement faible au Royaume-Uni (11) et en Nouvelle-Zélande (14), et relativement fort en France (15) et en Lituanie (16). II peut varier également selon les causes de décès (16). Certaines causes de décès présentent une connotation sociale (alcoolisme, suicide...) qui peut influer sur la présence de proches au moment du décès, ou leur déclaration à l'état civil.

Une autre limite de cette approche provient du fait que la dernière profession occupée avant le décès peut être influencée par le processus morbide ayant entraîné la mort: cette situation est particulièrement visible pour les sujets déclarés sans profession à l'état civil (15), caractéristique d'un effet de sélection $(11,14,17)$. Ainsi, le sens de la causalité reflétée par cette association est moins clairement établi (18). Ce phénomène peut être d'amplitude variable selon la cause de décès considérée. En effet, certaines causes résultent de longues maladies, susceptibles d'engendrer un déclassement social sur le long terme. Cet effet de sélection est moins présent pour d'autres causes, telles que certaines morts violentes ou maladies à développement très rapide. 
Les mesures d'inégalités de santé à l'aide de données chaînées permettent de s'affranchir du biais "Numérateur/Dénominateur", la même information étant utilisée au numérateur et au dénominateur. C'est pour cette raison que cette méthode a été utilisée à partir des années 1960, notamment en France $(19,20)$ et au Royaume-Uni (21). En France, cette approche a permis de mettre en évidence d'importants écarts sociaux de mortalité toutes causes de décès confondues $(13,22,23)$, et par cause de décès (24-31). Bien que des effets de sélection (11), ou de perte de vue sélectives de sujets des cohortes considérées (32) puissent limiter son interprétation, cette approche méthodologique est aujourd'hui considérée comme la référence (6). Cependant, dans la majorité des pays dont la France, pour des raisons de coûts, les effectifs de ces cohortes demeurent le plus souvent insuffisants pour permettre une analyse fine des différentiels de mortalité pour certaines causes spécifiques de décès (33), comme par exemple l'évolution récente de la mortalité par suicide en France selon la PCS (25).

En France, la demande sociale et institutionnelle de productions régulières d'études traitant des différentiels socio-économiques de mortalité par cause est forte. Parallèlement, la disponibilité de données longitudinales chaînées, représentatives de la population française et appariées aux causes de décès a longtemps été impossible pour des raisons de secret statistique ou médical (3). Dans ce contexte, certaines études ont adopté des méthodologies pragmatiques et approximatives: application d'une règle de trois pour déterminer les différentiels de mortalité par cause (13) ou utilisation de données non chaînées aussi bien en France $(9,34-36)$ que pour des comparaisons internationales (37). Malgré les limites de ces approches $(10,38)$, certains résultats sont repris dans le débat public sans précaution particulière. Par ailleurs, depuis 1999, la réforme de la statistique d'état civil par l'INSEE a modifié les règles de recueil et de codage de la PCS. La PCS n'est plus renseignée pour les sujets retraités dans les données d'état civil, et seules les données des classes d'âge actif, de 25 à 55 ans sont potentiellement exploitables. La définition de la catégorie des inactifs a 
pu évoluer suite à ce changement, alors même que cette catégorie participe fortement aux inégalités de mortalité (31).

La présente étude se propose de présenter et discuter l'état actuel des données disponibles permettant d'évaluer les différentiels socio-économiques de mortalité par cause en France dans les populations âgées de 25 à 55 ans.

Le premier objectif consiste à caractériser les écarts de classement entre les PCS déclarées au moment du recensement et celles issues de la déclaration à l'état civil au moment du décès, à partir des données de l'EDP (Echantillon Démographique Permanent). Les données de la cohorte EDP sont à ce jour les seules données disponibles et représentatives de la population française permettant de comparer la PCS déclarée à l'état civil au moment du décès à celle déclarée lors du dernier recensement précédant le décès.

Le second objectif consiste à évaluer les écarts d'estimation de risque relatif de mortalité par cause entre l'approche non chaînée et l'approche chaînée. Ces écarts seront caractérisés selon le sexe et la cause de décès pour les recensements de 1982, 1990 et 1999. Cette évaluation n'a pas été faite récemment (10), et n'a jamais été faite par cause de décès en France (16). Les données non chaînées utilisées sont issues de la base nationale exhaustives des causes de décès, et les données chaînées sont issues de la cohorte EDP.

Cette analyse permet de formuler des recommandations sur l'usage de la PCS issue de la base de données sur les causes de décès dans les études utilisant des données non chaînées, et sur les pistes d'améliorations de la statistique française pour permettre une estimation non biaisée, précise et interprétable des différentiels socio-économiques de mortalité par cause.

\section{Matériel et méthodes}

Sources des données 
Approche non chaînée : données exhaustives de mortalité par cause de décès

Pour chaque décès survenant en France, un certificat médical doit être rédigé par un médecin afin d'autoriser la fermeture du cercueil. Ce certificat médical est accompagné d'un bulletin d'état civil rédigé par un agent dans la mairie de survenue du décès sur la base des déclarations de la famille ou des proches (39). La profession en clair mentionnée sur le bulletin d'état civil est ensuite codée en PCS. Au moment de la refonte des statistiques de l'état-civil à la fin des années 90 , le questionnement sur la profession a été limité aux personnes actives au moment du décès, alors qu'elle était théoriquement déclarée systématiquement avant cette période.

Le fichier des données harmonisées des recensements de la population de 1968 à 2008 de I'INSEE a été utilisé pour estimer les proportions de population née en France Métropolitaine par PCS, selon l'âge et le sexe. Ces proportions ont par la suite été interpolées et multipliées par les estimations nationales, fournies par l'INSEE, de population par âge et sexe au $1^{\text {er }}$ janvier de chaque année.

Approche chaînée : Echantillon Démographique Permanent (EDP)

L'Echantillon Démographique Permanent de I'INSEE est un panel de près de $1 \%$ de la population française constitué de tous les individus nés entre les $1^{\text {er }}$ et 4 octobre de chaque année. Pour chacun de ces individus, les informations collectées à chaque recensement, de 1968 à 1999, sont chaînées. Ce panel est représentatif de la population française, dans la mesure où la date de naissance n'intervient pas sur le phénomène étudié.

Dans le cadre du projet EDISC (31), un appariement a été effectué pour les sujets décédés issus de ce panel avec les données de mortalité par causes médicales de décès produites par l'Inserm-CépiDc. Le taux d'appariement entre les deux bases est supérieur à $98 \%$. Compte tenu du taux important de perdus de vue parmi les sujets nés hors de France Métropolitaine, ceux-ci ont été exclus de la base de données. Les causes de décès et toutes les informations présentes sur le certificat ou le bulletin d'état civil de décès, dont la PCS 
déclarée à l'état civil au moment du décès, enrichissent les informations recueillies lors des recensements successifs pour les sujets de l'EDP.

\section{Catégories considérées}

Afin de ne considérer que les sujets majoritairement en âge d'être actifs, les analyses sont limitées aux personnes de 25 à 55 ans.

En raison d'un fort taux de perdu de vue des sujets nés hors de la France Métropolitaine dans l'EDP, l'ensemble des analyses a été limité aux sujets nés en France métropolitaine.

Trois périodes ont été considérées, relativement aux recensements de 1982, 1990 et 1999 : 1983-1989, 1991-1997 et 2000-2006. L'année du recensement a été exclue des analyses pour limiter les effets de sélection liée à la santé sur la PCS déclarée au recensement (14).

Au cours du suivi, quatre versions successives de la classification des PCS ont été utilisées pour coder la PCS déclarée à l'état civil (PCSEC). Les PCS déclarées aux différents recensements (PCSRE) ont toutes été recodées avec la même classification. Des regroupements de PCS larges ont été considérés dans cette analyse, en prenant en compte uniquement le premier caractère des PCS renseignées, à l'exception de l'année 1983 pour la PCSEC. Les différentes versions de la classification des PCS ont été harmonisées en 7 classes. L'ensemble de ces regroupements est résumé dans le tableau 1. La catégorie "7. retraités" constitue un groupe alors que les informations disponibles dans l'EDP auraient pu permettre de reclasser les sujets en fonction de leur précédente PCS. Ce choix a été fait pour maximiser la comparabilité entre données d'état civil et données de l'EDP. Cependant, pour les tranches d'âges considérées dans cette étude, le nombre de décès des retraités est très faible, et a dû être regroupé avec la catégorie des inactifs.

Tableau 1 ici 
Cause de décès

Les causes de décès sont codées à l'aide de deux versions de la classification internationale des maladies (CIM), en CIM9 de 1983 à 1997 puis en CIM10 à partir de 2000. Plusieurs grandes catégories de causes de décès ont été constituées et étudiées séparément: maladies liées à la consommation d'alcool (cancer des voix aérodigestives supérieures (Codes CIM9 : 140-149, CIM10 : C00-C14), psychoses alcoolique et alcoolisme (CIM9 : 291, 303, CIM10 : F10), cirrhose alcoolique ou sans précision du foie (Codes CIM9: 571.0-3, 571.5, CIM10: K70, K74.6)), cancers à l'exception des cancers des voies aérodigestives supérieures (CIM9 : 140-239, CIM10 : C15-D48), maladies infectieuses (CIM9 : 001-139, CIM10 : A00-B99), maladies cardiovasculaires (CIM9 : 390-459, CIM10 : I00-199), suicides (CIM9: E950-E959, CIM10: X60-X84) et autres causes externes (CIM9: E800-E999, CIM10 : V01-Y89 à l'exception des suicides). 


\section{Comparaison deux à deux des PCS à partir des données de l'EDP}

La caractérisation des écarts de classement entre les PCS déclarées au recensement et à l'état civil pour un même individu a été effectuée à l'aide des mesures suivantes :

- pourcentage de décès classés de façon identique au recensement et à l'état civil parmi les décès dont la PCS est renseignée à l'état civil,

- pourcentage de décès classés de façon identique au recensement et à l'état civil parmi les décès dont la PCS est renseignée au recensement,

- pour chaque catégorie de PCS, les ratios du total des décès déclarés au recensement (PCSRE) sur le total des décès déclarés à l'état civil (PCSEC). Ces ratios seront nommés "ratios des effectifs marginaux".

En raison des faibles effectifs et pour préserver la confidentialité des données présentées, toutes les combinaisons de PCSRE et PCSEC ne peuvent être présentées. Pour chaque PCSRE considérée, les PCSEC ont été regroupées en plusieurs catégories : la PCS identique, les PCS de numéro inférieur, les PCS de numéro supérieur, la PCS "inactif" et la PCS "non renseignée".

Comparaison des risques relatifs obtenus à partir des approches chaînées et non chaînées

Les approches chaînées et non chaînées, utilisant respectivement les données de l'EDP et les données exhaustives d'état civil, sont comparées en calculant des risques de mortalité relatifs au risque de mortalité des cadres et professions intellectuelles supérieures.

Les populations âgées entre 25 et 49 ans en début de période d'observation ont été sélectionnées.

Pour l'approche non chaînée, afin de maximiser la comparabilité avec l'approche chaînée, des quotients de mortalité sont calculés chaque année par cohorte de naissance de 5 ans, par sexe et par PCS. 
Pour l'approche chaînée, des quotients de mortalité sont calculés sur l'ensemble des périodes par cohorte de naissance de 5 ans, par sexe et par PCS.

Des modèles de Poisson spécifiques à chaque période, sexe et cause sont utilisés pour estimer les risques relatifs de mortalité selon la PCS relativement aux cadres et professions intellectuelles supérieures. Ces modèles sont ajustés sur la cohorte de naissance prise comme variable catégorielle et ne prennent pas en compte la surdispersion des données. Ainsi, les intervalles de confiance à $95 \%$ calculés ne dépendent que des effectifs présents, et non de la qualité d'ajustement du modèle employé (40).

En ajustant sur l'âge de façon identique entre les deux approches, les analyses faites à partir des données non chaînées font l'hypothèse que la déformation au cours du temps de la distribution par âge des populations est similaire selon les différentes PCS. Une analyse de sensibilité a été effectuée en introduisant une interaction entre l'année de suivie et la cohorte de naissance dans la modélisation des quotients calculés à partir des données non chaînées. Les résultats n'étaient pas modifiés.

\section{Résultats}

\section{Distribution des décès par PCS selon les sources et les périodes}

Quelle que soit la période, le nombre de décès observé dans la cohorte EDP correspond à près de $1 \%$ du nombre de décès de la base exhaustive d'état civil (tableau 2).

Concernant les PCS retraités, inactifs et non renseigné, la proportion d'inactifs, plus élevée pour les femmes, est nettement inférieure dans les données de l'EDP par rapport aux données d'état civil, avec en particulier une forte augmentation de cette proportion à l'état civil durant la période 2000-2006. En revanche, la proportion de données manquantes est plus élevée dans l'EDP que dans les données d'état civil.

Concernant les PCS informant sur la profession, pour les hommes, les proportions de décès obtenues pour la PCS "5. Employés" sont similaires ou inférieures, tandis que les proportions 12 
des autres PCS sont systématiquement supérieures dans I'EDP comparées aux données d'état civil. Pour les femmes, les PCS, en proportion, sont quasi-systématiquement supérieures dans l'EDP par rapport aux données d'état civil.

Tableau 2 ici

\section{Comparaison des PCS déclarées au recensement et à l'état civil sur la période 2000-2006}

Le croisement de la PCSRE déclarée en 1999 avec la PCSEC met en évidence une concordance faible (tableau 3). Prises globalement, 49\% des PCSRE connues sont reportées de manière équivalente à l'état civil. Cette proportion est plus élevée pour les PCS agriculteurs-exploitants (64\%) et plus faible pour les artisans, commerçants et chefs d'entreprise et les cadres et professions intellectuelles supérieures (environ 32\%).

En prenant un autre angle, seules 54\% des PCSEC connues ont été déclarées de manière identique au précédent recensement. Cette proportion est répartie de façon identique pour les différentes PCS des actifs. En revanche, 36\% des PCSRE sont identiques (résultat non montré) lorsque la PCSEC est "8. Inactifs", cette proportion est bien moins élevée que la proportion de PCSEC identique lorsque que la PCSRE est "8. Inactifs", de 64\%

Les nombres de décès marginaux obtenus avec ces deux mesures sont d'un ordre de grandeur similaire pour les agriculteurs-exploitants et les employés (ratios entre marges PCSRE/PCSEC respectivement de 1,00 et 1,06 ) tandis que pour les autres PCS, et en particulier les artisans, commerçants et chefs d'entreprise et les cadres et professions intellectuelles supérieures (PCSRE/PCSEC respectivement de 1,38 et 1,36) les effectifs de décès sont sensiblement plus élevés en considérant la PCS au recensement par rapport à la PCS déclarée à l'état civil. 
On peut discerner derrière ces différentiels une tendance au déclassement social en passant de la déclaration au recensement à celle de l'état-civil. C'est particulièrement visible pour les sujets déclarant une profession intermédiaire au recensement, et pour lesquels la PCS reportée à l'état civil est classée "5. Employés" ou "6. Ouvriers" dans 50\% des cas contre agriculteurs, artisans, et cadres dans seulement $6 \%$ des cas.

Les PCS d'actifs au recensement accompagnées le plus fréquemment d'une PCS "8. Inactif" à l'état civil sont les employés et ouvriers (27\% et $30 \%$ respectivement).

Tableau 3 ici

\section{Comparaison du risque relatif de mortalité selon l'approche}

Résultats par PCS selon la période pour la mortalité toutes causes

Pour les hommes, les risques relatifs estimés en utilisant l'approche non chaînée sont nettement supérieurs à ceux estimés à partir de l'approche chaînée pour toutes les PCS sur la période 2000-2006 et pour les PCS "artisans, commerçants" et "employés" sur toutes les périodes (tableau 4). En revanche, les risques relatifs obtenus selon les deux approches pour les PCS "ouvriers", "professions intermédiaires" et "inactifs" sont proches pour les deux premières périodes. Les risques relatifs obtenus pour cette dernière catégorie sont très élevés, proche de 10 sur les périodes 1983-1989 et 1991-1997, et proche de 20 en considérant l'approche non chainée sur la période 2000-2006 (RR : 21,64 [21,15; 22,14]).

Quelles que soient la période et la cause de décès considérées, les intervalles de confiance des risques relatifs issus de l'approche chaînée sont plus larges que ceux issus de l'approche non chaînée du fait de la différence d'effectifs. Pour les femmes, à l'exception de la catégorie des inactives, aucun des intervalles de confiance n'est disjoint entre les deux sources d'information. 
Tableau 4 ici

Comparaison des ouvriers avec les cadres selon la période et la cause de décès

Durant les périodes 1983-1989 et 1991-1997, quelle que soit l'approche, les risques relatifs sont particulièrement élevés pour les causes liées à l'alcool et proches du risque relatif de mortalité toutes causes pour les autres causes considérées à l'exception des maladies infectieuses pour lesquelles les risques relatifs sont inférieurs à 1 (tableau 5). Sur ces deux périodes, les risques relatifs sont d'un ordre de grandeur similaire selon les deux approches. Cette similarité est particulièrement observable pour les hommes, avec toutefois des risques relatifs sensiblement plus élevés pour les cancers quand on compare l'approche non chaînée et l'approche chaînée. Sur la dernière période, en revanche, les risques relatifs pour les hommes sont systématiquement supérieurs en considérant l'approche non chaînée comparée à l'approche chaînée, même s'ils y a peu d'intervalles de confiance disjoints entre les deux estimations.

Pour certaines causes, et plus particulièrement chez les femmes, les effectifs sont trop faibles pour permettre une estimation du risque relatif pour la tranche d'âge considérée dans cette étude (25-55 ans) à partir des données de l'EDP.

Tableau 5 ici

Résultats par PCS sur la période 2000-2006 chez les hommes selon la cause

Bien que rarement significativement différentes, les estimations de risques relatifs faites à l'aide des données chaînées de l'EDP sont systématiquement inférieures à celles obtenues avec les données non chaînées d'état civil (tableau 6). La position relative des risques relatifs par PCS est sensiblement identique selon les deux approches, chaînée et non chaînée. La mortalité est généralement plus élevée pour les ouvriers et employés, suivie de 
celle des agriculteurs exploitants, puis de celles, proches, des artisans, commerçants et chefs d'entreprise et des professions intermédiaires. Cet ordre est modifié de façon identique pour les deux approches pour les suicides. Les risques relatifs les plus élevés sont alors obtenus pour les agriculteurs exploitants suivi des employés et ouvriers.

Les risques relatifs estimés pour la catégorie des inactifs sont très élevés pour les deux approches, particulièrement pour les hommes en utilisant l'approche non chaînée.

Tableau 6 ici 


\section{Discussion}

En considérant des catégories homogènes de PCS, les distributions des décès par PCS varient sensiblement selon la source de données (état civil ou recensement précédent). En particulier, les proportions d'employés et d'inactifs sont plus importantes dans les données d'état civil. Sur la période 2000-2006, la concordance est faible entre la PCS déclarée lors du recensement précédent et celle déclarée à l'état civil : seule la moitié des décès concorde entre les deux sources. Les écarts sociaux de mortalité sont généralement plus élevés avec l'approche non chaînée, en particulier entre les employés et les cadres. Les risques relatifs chez les ouvriers (par rapport aux cadres) sont similaires selon les deux approches sur les périodes 1983-1989 et 1991-1997 et plus élevés pour l'approche non chaînée sur la période 2000-2006. Sur cette dernière période, l'ordre et l'amplitude des risques relatifs varient de façon similaire selon la PCS et la cause de décès pour les deux approches. Par ailleurs, quelles que soient la période et la cause considérées, les intervalles de confiance des estimations obtenues à partir de l'approche chaînée sont plus larges que ceux de l'approche non chaînée.

Ces résultats confirment que les biais numérateur-dénominateur des études basées sur les données d'état civil sont importants en France, comme cela avec été montré dans des études précédentes $(10,15)$. En particulier, la catégorie des employés est nettement surreprésentée à l'état civil sur les périodes précédant 1999, mettant en évidence l'influence du mode de recueil sur la PCS recueillie (41). Cependant, et par le fait d'une compensation probablement fortuite, les résultats concernant la comparaison entre les ouvriers et les cadres ne sont pas fortement influencés par le choix de l'approche avant 1999, aussi bien pour la mortalité toutes causes que par cause.

Outre le biais numérateur-dénominateur, l'évolution de la situation socioprofessionnelle de l'individu entre le moment du recensement et celui du décès constitue un autre facteur 
associé à la faible concordance entre les deux sources. Cette évolution peut être soit une hausse graduelle dans l'échelle sociale attribuable à une avancée dans la carrière, soit un changement de position socioéconomique attribuable à l'état de santé de la personne dans la période précédant le décès. La comparaison des deux PCS déclarées semble indiquer que la PCS est plus souvent dégradée à l'état-civil par rapport au recensement, suggérant que l'état de santé ait pu jouer un rôle dans cette dégradation. II en résulte d'une part que des résultats fondés sur les données d'état civil auront tendance à aboutir à des différentiels sociaux de mortalité supérieurs aux résultats obtenus à partir de données chaînées, et d'autre part que le sens de l'association entre PCS et mortalité est moins aisément interprétable.

L'analyse par cause de décès indique que les différences de risques relatifs entre les deux approches ne sont pas particulièrement sensibles à la cause de décès considérée. Cependant, la faible puissance des résultats de l'approche chaînée limite l'analyse des écarts entre les deux approches par cause.

Une manière de renforcer la puissance et d'améliorer la comparabilité des différentes périodes considérées, consiste à calculer un indicateur synthétique des inégalités de mortalité, I'Indice Relatif d'Inégalité (42). Toutefois, cette méthode ne peut être appliquée que dans la mesure où les catégories sociales sont ordonnées à partir de considération a priori (43), ce qui n'est pas forcément le cas pour les PCS. De plus, les estimations par PCS spécifique demeurent en elles-mêmes utiles pour permettre d'identifier des déterminants socio-professionnels spécifiques pour certaines causes de décès.

La grande variabilité observée dans le pourcentage de PCS "non déclaré" ou "inactifs" selon les sources et la période considérée rend difficile l'analyse de l'évolution globale des inégalités sociales de mortalité. Des méthodes ont été proposées pour corriger ces éventuels biais, basées sur la redistribution des inactifs au prorata des dernières PCS déclarées avant l'inactivité à partir d'autres jeux de données $(44,45)$. Cependant, compte- 
tenu de la distribution différente de la non-réponse et de l'inactivité entre le recensement et l'état civil, ces méthodes de correction appliquées à cette analyse auraient nécessité de faire de nombreuses hypothèses. Ces méthodes n'ont pas été retenues dans cette analyse.

Dans un souci de comparabilité des résultats obtenus avec les deux approches et sur les différentes périodes, cette étude considère les classes d'âges entre 25 et 55 ans, (représentant $9 \%$ du total des décès). Les différentiels obtenus pour ces classes d'âges ne sont pas représentatifs des différentiels de mortalité dans leur globalité. Cependant, les différentiels socioéconomiques de mortalité à ces âges sont généralement particulièrement élevés $(46,47)$. La mortalité entre 25 et 55 ans, correspondant à des décès précoces pouvant être évités par des actions ciblées et ayant une forte répercussion sociale et économique, représente un enjeu de santé publique important.

Les écarts d'estimations qui seraient obtenus entre les deux approches en prenant en compte des classes d'âges plus élevés seraient probablement différents. En effet, d'une part, des erreurs de retranscription de l'ancienne profession par les proches sont susceptibles d'être plus fréquentes lorsque le délai entre la fin d'activité et le décès augmente. D'autre part, pour les sujets partis à la retraite, la dernière profession occupée est moins influencée par le processus morbide conduisant au décès.

Les deux sources utilisées présentent des limites. L'usage de la PCS déclarée à l'état civil lors du décès est à l'origine de biais importants pour des études épidémiologiques et de santé publique, y compris pour les classes d'âges où la proportion de population active est élevée. Les données de l'EDP sont à ce jour de taille insuffisante pour une étude fine par cause de décès. En l'absence d'autres informations, ces deux sources de données doivent ainsi être utilisées avec précautions pour documenter les inégalités sociales de mortalité. 
Une amélioration du recueil de la PCS à l'état civil ou de ses modalités de codage pourrait diminuer certains biais, mais ne semble pas pouvoir les éliminer de façon satisfaisante pour un usage rigoureux, d'autant plus que la déclaration à l'état civil d'un décès ne peut pas faire l'objet de questions précises dans un certain nombre de situations.

Dans ce contexte, une des options en vue d'améliorer la statistique d'état civil consisterait à recueillir le niveau d'études à la place ou en plus de la PCS lors de la déclaration à l'état civil. Le niveau d'études est moins sujet à interprétation. II est généralement fixé tôt dans la vie et n'est pas influencé par l'évolution de l'état de santé ultérieur (48). Cependant, cette variable peut également être entachée de biais de déclaration important et d'amplitude variable selon les pays, certaines études retrouvant de larges écarts de déclaration entre la déclaration à l'état civil au moment du décès et au précédent recensement $(49,50)$, d'autres des écarts plus réduits (51).

Les résultats obtenus nous poussent principalement à préconiser des appariements à visée exhaustive, ou tout du moins de plus grande taille que l'EDP, entre les données de mortalité par cause de décès et des données sociales issues de différentes bases nationales telles que le recensement, les bases des caisses d'assurance-vieillesse (52) ou les bases issues des déclarations de revenu aux impôts (53). De tels appariements sont aujourd'hui techniquement réalisables en respectant les règles de confidentialité des données. Ils apporteraient la possibilité d'études fines de l'évolution des disparités de mortalité par cause en population générale. 


\section{Références}

1. Leclerc A, Kaminski M, Lang T. Inégaux face à la santé. Paris: La Découverte; 2008.

2. Lang T, Badeyan G, Cases C, Chauvin P, Duriez M, Grémy I, et al. Les inégalités sociales de santé : sortir de la fatalité. Paris: Haut Conseil de la Santé Publique, 2009.

3. Goldberg M, Cases C, Desenclos JC, Jougla E, Leclerc A, Merlière Y, et al. Les systèmes d'information pour la santé publique. Paris: Haut Conseil de la Santé Publique, 2009.

4. Ribet C, Melchior M, Lang T, Zins M, Goldberg M, Leclerc A. [Characterisation and measurement of social position in epidemiologic studies]. Rev Epidemiol Sante Publique. 2007;55:285-95.

5. Loi relative à la politique de santé publique, Assemblée nationale, 9 août 2004.

6. Valkonen T. Les inégalités sociales devant la mort. In: Caselli G, Vallin J, Wunsch G, eds. Démographie : analyse et synthèse, volume 3 : Les déterminants de la mortalité. Paris: INED; 2002.

7. Huber M. La mortalité suivant la profession. Bulletin de la statistique générale de la France. 1911-1912:402-39.

8. Febvay M, Aubenque M. La mortalité par catégorie socio-professionnelle. Etudes statistiques. 1957;3.

9. Bouvier-Colle MH, Barberena A, Hatton F. [Mortality by socio-occupational categories in urban and rural areas]. Rev Epidemiol Sante Publique. 1983;31:115-28.

10. Kunst AE, Groenhof F, Borgan JK, Costa G, Desplanques G, Faggiano F, et al. Socioeconomic inequalities in mortality. Methodological problems illustrated with three examples from Europe. Rev Epidemiol Sante Publique. 1998;46:467-79. 
11. Fox AJ, Goldblatt PO, Jones DR. Social class mortality differentials: artefact, selection or life circumstances? J Epidemiol Community Health. 1985;39:1-8.

12. Ogle W. Letter to the Registrar General on the mortality in the registration districts of England and Wales during the ten years 1871-1880. Supplement to the 45th Annual Report of the Registrar General of Births, Deaths and Marriages in England. London: Registrar general; 1885.

13. Desplanques G. L'inégalité sociale devant la mort. In: Bouvier-Colle MH, Vallin J, Hatton F, eds. Mortalité et causes de décès en France. Paris: INSERM; 1990. p. 257-87.

14. Blakely $\mathrm{T}$, Fawcett $\mathrm{J}$. Bias measuring mortality gradients by occupational class in New Zealand. N Z Med J. 2005;118:U1253.

15. Lévy C, Vallin J. La mortalité par catégorie socio-professionnelle. Un essai de calcul direct. Population. 1981;4-5:938-45.

16. Shkolnikov VM, Jasilionis D, Andreev EM, Jdanov DA, Stankuniene V, Ambrozaitiene D. Linked versus unlinked estimates of mortality and length of life by education and marital status: evidence from the first record linkage study in Lithuania. Soc Sci Med. 2007;64:1392406.

17. Fox AJ, Goldblatt PO, Adelstein AM. Selection and mortality differentials. J Epidemiol Community Health. 1982;36:69-79.

18. Krieger N, Williams DR, Moss NE. Measuring social class in US public health research: concepts, methodologies, and guidelines. Annu Rev Public Health. 1997;18:341-78.

19. Calot G, Febvay M. La mortalité différentielle suivant le milieu social. Présentation d'une méthode expérimentée en France sur la période 1955-1960. Premiers résultats. Etudes et conjoncture. 1965;11:75-160. 
20. Desplanques G. A 35 ans, les instituteurs ont encore 41 ans à vivre, les manoeuvres 34 ans seulement. Economie et Statistiques. 1973;49:3-19.

21. Fox AJ, Goldblatt PO. Longitudinal study: socio-demographic mortality differentials a first report on mortality in 1971-1975 according to 1971 census characteristics, based on data collected in the OPCS Longitudinal Study. London: Office of Population Censuses and Surveys, 1982.

22. Monteil C, Robert-Bobée I. Les différences sociales de mortalité : en augmentation chez les hommes, stables chez les femmes. INSEE Première. 2005.

23. Blanpain $\mathrm{N}$, Chardon $\mathrm{O}$. Les inégalités sociales face à la mort. Tables de mortalité par catégorie sociale et indices standardisés de mortalité pour quatre périodes (1976-1984, 1983-1991, 1991-1999, 2000-2008): INSEE, Direction des statistiques démographiques et sociales, 2011.

24. Saurel-Cubizolles MJ, Chastang JF, Menvielle G, Leclerc A, Luce D. Social inequalities in mortality by cause among men and women in France. J Epidemiol Community Health. 2009;63:197-202.

25. Cohidon C, Santin G, Geoffroy-Perez B, Imbernon E. [Suicide and occupation in France]. Rev Epidemiol Sante Publique. 2010;58:139-50.

26. Menvielle G, Leclerc A, Chastang JF, Melchior M, Luce D. Changes in socioeconomic inequalities in cancer mortality rates among French men between 1968 and 1996. Am J Public Health. 2007;97:2082-7.

27. Menvielle G, Chastang JF, Luce D, Leclerc A. [Changing social disparities and mortality in France (1968-1996): cause of death analysis by educational level]. Rev Epidemiol Sante Publique. 2007;55:97-105. 
28. Menvielle G, Luce D, Geoffroy-Perez B, Chastang JF, Leclerc A. Social inequalities and cancer mortality in France, 1975-1990. Cancer Causes Control. 2005;16:501-13.

29. Menvielle G, Leclerc A, Chastang JF, Luce D. Social inequalities in breast cancer mortality among French women: disappearing educational disparities from 1968 to $1996 . \mathrm{Br} \mathrm{J}$ Cancer. 2006;94:152-5.

30. Menvielle G, Leclerc A, Chastang JF, Luce D. Socioeconomic inequalities in cause specific mortality among older people in France. BMC Public Health. 2010;10:260.

31. Leclerc A, Chastang JF, Menvielle G, Luce D. Socioeconomic inequalities in premature mortality in France: have they widened in recent decades? Soc Sci Med. 2006;62:2035-45.

32. Geoffroy-Perez B. Analyse de la mortalité et des causes de décès par secteur d'activité de 1968 à 1999 à partir de l'Echantillon démographique permanent : Etude pour la mise en place du programme Cosmop : Cohorte pour la surveillance de la mortalité par profession. Saint-Maurice: InVS, 2006.

33. Fox AJ. Socio-demographic mortality differentials: new longitudinal perspectives. Rev Epidemiol Sante Publique. 1987;35:20-7.

34. Jougla E, Rican S, Péquignot F, Le Toullec A. Disparités sociales de mortalité. Rev Prat. 2004;54:2228-32.

35. Rey G. Données de décès par suicide. In: Bonnin F, Chenu C, Duprez V, Etiemble J, Gomis C, Lecomte M-C, et al., eds. Expertise collective : Stress au travail et santé, situation chez les indépendants: Inserm; 2011.

36. Jougla E, Hirsch A. La durée de vie en France. Paris: Haut Comité de la Santé Publique, 1994. 
37. Kunst AE, Groenhof F, Mackenbach JP, Health EW. Occupational class and cause specific mortality in middle aged men in 11 European countries: comparison of population based studies. EU Working Group on Socioeconomic Inequalities in Health. Bmj. 1998;316:1636-42.

38. Valkonen T. Problems in the measurement and international comparisons of socioeconomic differences in mortality. Soc Sci Med. 1993;36:409-18.

39. Pavillon G, Laurent F. Certification et codification des causes médicales de décès. Bul Epidémiol Hebd. 2003;30-31:134-8.

40. McCullagh P, Nelder JA. Generalized Linear Models. London: Chapman and Hall; 1989.

41. Chenu A, Guglielmetti F. Coder la profession. Nouvelles procédures, vieux problèmes. Courrier des statistiques. 2000;95-96.

42. Mackenbach JP, Kunst AE. Measuring the magnitude of socio-economic inequalities in health: an overview of available measures illustrated with two examples from Europe. Soc Sci Med. 1997;44:757-71.

43. Regidor E. Measures of health inequalities: part 2. J Epidemiol Community Health. 2004;58:900-3.

44. Williams GM, Najman JM, Clavarino A. Correcting for numerator/denominator bias when assessing changing inequalities in occupational class mortality, Australia 1981 -2002. Bull World Health Organ. 2006;84:198-203.

45. Kunst AE, Groenhof F, Mackenbach JP. Mortality by occupational class among men 30-64 years in 11 European countries. EU Working Group on Socioeconomic Inequalities in Health. Soc Sci Med. 1998;46:1459-76. 
46. Merlo J, Gerdtham UG, Lynch J, Beckman A, Norlund A, Lithman T. Social inequalities in health- do they diminish with age? Revisiting the question in Sweden 1999. Int J Equity Health. 2003;2:2.

47. Rey G, Jougla E, Fouillet A, Hemon D. Ecological association between a deprivation index and mortality in France over the period 1997 - 2001: variations with spatial scale, degree of urbanicity, age, gender and cause of death. BMC Public Health. 2009;9:33.

48. Davey Smith G, Hart C, Hole D, MacKinnon P, Gillis C, Watt G, et al. Education and occupational social class: which is the more important indicator of mortality risk? J Epidemiol Community Health. 1998;52:153-60.

49. Sorlie PD, Johnson NJ. Validity of education information on the death certificate. Epidemiology. 1996;7:437-9.

50. Shai D, Rosenwaike I. Errors in reporting education on the death certificate: some findings for older male decedents from New York State and Utah. Am J Epidemiol. 1989;130:188-92.

51. Rosamond WD, Tyroler HA, Chambless LE, Folsom AR, Cooper L, Conwill D. Educational achievement recorded on certificates of death compared with self-report. Epidemiology. 1997;8:202-4.

52. Poubelle V, Albert C, Beurnier P, Couhin J, Grave N. Prisme, le modèle de la Cnav. Retraite et société. 2006;48:202-15.

53. Jusot F. Revenu et Mortalité : Analyse Economique des Inégalités Sociales de Santé en France. Paris: Ecole des Hautes Etudes en Sciences Sociales; 2003. 


\section{Tableaux}

Tableau 1. Regroupements de codes de PCS utilisés selon la source de données et la période étudiée

\begin{tabular}{|c|c|c|c|c|c|}
\hline & \multirow[t]{2}{*}{ Recensement (PCSRE) } & \multicolumn{4}{|c|}{ Etat civil (PCSEC) } \\
\hline & & 1983 & 1984-1987 & 1988-1998 & 1999-2007 \\
\hline 1. Agriculteurs exploitants & $10-13$ & 00 & 10 & 10 & $10-14$ \\
\hline $\begin{array}{l}\text { 2. Artisans, commerçants et } \\
\text { chefs d'entreprise }\end{array}$ & $21-23$ & $21-27$ & $21-23$ & $21-23$ & $21-23$ \\
\hline $\begin{array}{l}\text { 3. Cadres et professions } \\
\text { intellectuelles supérieures }\end{array}$ & $31-38$ & $30-34,80$ & $31-38$ & $31-36$ & $31-38$ \\
\hline 4. Professions intermédiaires & $42-48$ & $41-44,60,81$ & $42-48$ & $42-48$ & $42-48$ \\
\hline 5. Employés & $52-56$ & $51-53,70-72$ & $52-56$ & $52-56$ & $52-56$ \\
\hline 6. Ouvriers & $62-69$ & $10,61-68$ & $62-69$ & $60,64-69$ & $62-69$ \\
\hline 7. Retraités & $70-78$ & $93-96,98$ & 70 & 70 & \\
\hline 8. Inactifs* & $81-86$ & $82,91,92,99$ & $82,83,84$ & $82-84$ & \\
\hline
\end{tabular}


Tableau 2. Nombre de décès par période et proportion de décès par PCS - par sexe et âge

\section{N $\%$ par PCS}

\begin{tabular}{|c|c|c|c|c|c|c|c|c|c|c|c|c|c|}
\hline & Source & Période & Total & 1. & 2. & 3. & 4. & 5. & 6. & 7. & 8. & $\mathbf{x}$. & Total \\
\hline \multirow{6}{*}{ Hommes } & \multirow{3}{*}{ EDP } & 1983-1989 & 2116 & 4.2 & 5.5 & 6.2 & 11.3 & 9.6 & 39.2 & 0.5 & 10.4 & 13.1 & 100.0 \\
\hline & & $1991-1997$ & 2184 & 2.8 & 6.6 & 7.3 & 14.7 & 9.8 & 36.5 & 1.1 & 10.1 & 11.1 & 100.0 \\
\hline & & $2000-2006$ & 2004 & 2.6 & 5.3 & 6.1 & 13.4 & 12.0 & 37.2 & 0.6 & 12.7 & 10.0 & 100.0 \\
\hline & \multirow{3}{*}{ Etat-civil } & 1983-1989 & 197929 & 3.7 & 6.6 & 5.1 & 9.9 & 14.0 & 34.8 & 1.7 & 18.2 & 6.0 & 100.0 \\
\hline & & 1991-1997 & 207542 & 2.2 & 6.2 & 5.8 & 11.5 & 14.6 & 31.3 & 1.0 & 21.3 & 6.1 & 100.0 \\
\hline & & $2000-2006$ & 190887 & 2.1 & 4.1 & 4.4 & 9.9 & 11.0 & 33.3 & 1.1 & 29.7 & 4.5 & 100.0 \\
\hline \multirow{6}{*}{ Femmes } & \multirow{3}{*}{ EDP } & 1983-1989 & 812 & 2.1 & 3.9 & 3.2 & 8.3 & 21.3 & 6.8 & 1.6 & 42.6 & 10.2 & 100.0 \\
\hline & & $1991-1997$ & 884 & 2.0 & 3.5 & 4.0 & 11.4 & 28.9 & 10.2 & 0.7 & 32.2 & 7.1 & 100.0 \\
\hline & & $2000-2006$ & 932 & 1.4 & 2.7 & 4.2 & 11.9 & 30.4 & 9.4 & 1.0 & 32.4 & 6.7 & 100.0 \\
\hline & \multirow{3}{*}{ Etat-civil } & 1983-1989 & 82437 & 2.3 & 2.8 & 2.5 & 8.5 & 20.9 & 5.7 & 1.0 & 50.3 & 6.0 & 100.0 \\
\hline & & 1991-1997 & 84646 & 1.2 & 2.7 & 3.1 & 11.1 & 24.9 & 5.4 & 0.9 & 45.0 & 5.8 & 100.0 \\
\hline & & $2000-2006$ & 87013 & 0.7 & 1.8 & 3.1 & 11.0 & 25.6 & 7.6 & 0.9 & 45.0 & 4.3 & 100.0 \\
\hline
\end{tabular}

* : 1. Agriculteurs exploitants 2. Artisans, commerçants et chefs d'entreprise. 3. Cadres et professions intellectuelles supérieures. 4. Professions intermédiaires 5 . Employés 6. Ouvriers 7. Retraités 8 . Inactifs, chômeurs n'ayant jamais travaillé et autres x. Non renseigné 
Tableau 3. Nombre de décès par PCS déclaré à l'état civil (PCSEC) et lors du recensement de 1999 (PCSRE) - 2000-2006 - sujets âgés de 25 à 49 ans au 11 janvier 1999 (Données $E D P)$

\begin{tabular}{|c|c|c|c|c|c|c|c|c|c|c|}
\hline $\mathbf{N}$ & \multicolumn{9}{|c|}{ PCS déclarée à l'état civil (PCSEC) } & \multirow{2}{*}{$\begin{array}{c}\text { Ratio } \\
\text { PCSRE/PCSEC }\end{array}$} \\
\hline $\begin{array}{l}\text { PCS déclarée au recensement } \\
\text { (PCSRE) }\end{array}$ & 1 & 2 & 3 & 4 & 5 & 6 & 8 & X. & Total & \\
\hline 1. Agriculteurs exploitants & $\begin{array}{r}42 \\
63.6\end{array}$ & & & $\begin{array}{c}13 \\
19.7\end{array}$ & & & - & $\begin{array}{l}- \\
-\end{array}$ & $\begin{array}{r}66 \\
100.0\end{array}$ & $1.00[0.82 ; 1.23]$ \\
\hline $\begin{array}{l}\text { 2. Artisans, commerçants et } \\
\text { chefs d'entreprise }\end{array}$ & & $\begin{array}{r}42 \\
31.8\end{array}$ & & & & & $\begin{array}{r}32 \\
24.2\end{array}$ & $\begin{array}{l}- \\
-\end{array}$ & $\begin{array}{r}132 \\
100.0\end{array}$ & $1.38[1.12 ; 1.69]$ \\
\hline $\begin{array}{l}\text { 3. Cadres et professions } \\
\text { intellectuelles supérieures }\end{array}$ & & & $\begin{array}{r}52 \\
32.3\end{array}$ & & $\begin{array}{c}65 \\
40.4\end{array}$ & & - & - & $\begin{array}{r}161 \\
100.0\end{array}$ & $1.36[1.13 ; 1.64]$ \\
\hline 4. Professions intermédiaires & & $\begin{array}{l}24 \\
6.3\end{array}$ & & $\begin{array}{r}140 \\
36.8\end{array}$ & & & $\begin{array}{r}66 \\
17.4\end{array}$ & $\begin{array}{l}27 \\
7.1\end{array}$ & $\begin{array}{r}380 \\
100.0\end{array}$ & $1.32[1.18 ; 1.48]$ \\
\hline 5. Employés & & 9 & & & $\begin{aligned} 239 \\
45.6\end{aligned}$ & $\begin{array}{c}59 \\
11.3\end{array}$ & $\begin{array}{r}143 \\
27.3\end{array}$ & $\begin{array}{r}32 \\
6.1\end{array}$ & $\begin{array}{r}524 \\
100.0\end{array}$ & $1.06[0.98 ; 1.16]$ \\
\hline 6. Ouvriers & & & $\begin{array}{l}128 \\
15.4\end{array}$ & & & $\begin{array}{r}413 \\
49.6\end{array}$ & $\begin{array}{r}251 \\
30.1\end{array}$ & $\begin{array}{l}41 \\
4.9\end{array}$ & $\begin{array}{r}833 \\
100.0\end{array}$ & $1.17[1.10 ; 1.24]$ \\
\hline 8. Inactifs* & & & $\begin{array}{c}1 \varepsilon \\
32\end{array}$ & & & & $\begin{array}{r}368 \\
63.8\end{array}$ & $\begin{array}{r}23 \\
4.0\end{array}$ & $\begin{array}{r}577 \\
99.0\end{array}$ & $0.57[0.53 ; 0.61]$ \\
\hline x. Non renseigné & & & $\begin{array}{c}12 \\
53\end{array}$ & & & & $\begin{array}{r}116 \\
44.1\end{array}$ & $\begin{array}{r}7 \\
2.7\end{array}$ & $\begin{array}{r}263 \\
100.0\end{array}$ & $1.73[1.42 ; 2.10]$ \\
\hline Total & $\begin{array}{r}66 \\
2.2\end{array}$ & $\begin{array}{r}96 \\
3.3\end{array}$ & $\begin{array}{l}118 \\
4.0\end{array}$ & $\begin{array}{r}287 \\
9.8\end{array}$ & $\begin{array}{r}493 \\
16.8\end{array}$ & $\begin{array}{r}714 \\
24.3\end{array}$ & $\begin{array}{r}1010 \\
34.4\end{array}$ & $\begin{array}{r}152 \\
5.2\end{array}$ & $\begin{array}{c}2936 \\
100.0\end{array}$ & \\
\hline
\end{tabular}

* : Inactifs, chômeurs n'ayant jamais travaillé, retraités et autres

- : effectifs trop faibles pour assurer la confidentialité des données

Note de lecture :

La PCSRE du défunt a été déclarée "4. Professions intermédiaires" pour 380 décès. Parmi ceux-ci, 24 décès $(6,3 \%)$, ont pour PCSEC "1. Agriculteurs exploitants", "2. Artisans, com. et chefs d'ent." ou "3. Cadres et prof. Int. Sup.", 140 décès $(36,8 \%)$ ont une PCSEC identique à la PCSRE, 123 décès $(32,4 \%)$ ont pour PCSEC " 5 . Employés" ou "Ouvriers", 66 décès $(27,3 \%)$ ont pour PCSEC "Inactifs" et $27(7,1 \%)$ n'ont pas de PCSEC renseignée.

Le ratio du nombre total de décès pour lesquels la PCSRE a été déclarée "5. Employés" (524), sur le nombre total de décès pour lesquels la PCSEC a été déclarée "5. Employés" (493), est égal à 1,06. 
Tableau 4. Risque relatif de mortalité par PCS vs. Cadres et professions intellectuelles supérieures, selon la source de données - par sexe et période

\begin{tabular}{|c|c|c|c|c|c|c|c|c|c|}
\hline \multirow[b]{2}{*}{ Période } & \multirow[b]{2}{*}{ PCS } & \multicolumn{4}{|c|}{ Homme } & \multicolumn{4}{|c|}{ Femme } \\
\hline & & \multicolumn{2}{|c|}{$\begin{array}{c}\text { Approche chaînée } \\
\text { (EDP) }\end{array}$} & \multicolumn{2}{|c|}{$\begin{array}{c}\text { Approche non } \\
\text { chaînée (Etat-civil) }\end{array}$} & \multicolumn{2}{|c|}{$\begin{array}{c}\text { Approche chaînée } \\
\text { (EDP) }\end{array}$} & \multicolumn{2}{|c|}{$\begin{array}{c}\text { Approche non } \\
\text { chaînée (Etat-civil) }\end{array}$} \\
\hline \multirow{6}{*}{$1983-1989$} & 1. Agriculteurs exploitants & 1.21 & {$[0.90 ; 1.64]$} & 1.75 & {$[1.69 ; 1.80]$} & 0.74 & {$[0.38 ; 1.45]$} & 1.12 & {$[1.05 ; 1.20]$} \\
\hline & 2. Artisans, com. et chefs d'ent. ${ }^{*}$ & 1.22 & {$[0.94 ; 1.59]$} & 1.76 & {$[1.72 ; 1.81]$} & 1.10 & {$[0.60 ; 2.02]$} & 1.09 & {$[1.02 ; 1.15]$} \\
\hline & 4. Professions intermédiaires & 1.13 & {$[0.90 ; 1.42]$} & 1.40 & {$[1.36 ; 1.43]$} & 0.88 & {$[0.52 ; 1.50]$} & 1.28 & {$[1.21 ; 1.34]$} \\
\hline & 5. Employés & 1.91 & {$[1.51 ; 2.42]$} & 4.23 & {$[4.13 ; 4.32]$} & 1.09 & {$[0.67 ; 1.77]$} & 1.43 & {$[1.36 ; 1.49]$} \\
\hline & 6. Ouvriers & 2.42 & {$[1.99 ; 2.94]$} & 2.85 & {$[2.79 ; 2.91]$} & 0.93 & {$[0.54 ; 1.62]$} & 1.18 & {$[1.12 ; 1.25]$} \\
\hline & 8. Inac & 9.30 & {$[7.40 ; 11.70]$} & 11.36 & {$[11.11 ; 11.61]$} & 1.72 & {$[1.07 ; 2.77]$} & 3.19 & {$[3.05 ; 3.33]$} \\
\hline \multirow{6}{*}{$1991-1997$} & 1. $A c$ & 1.34 & {$[0.98 ; 1.83]$} & 1.45 & {$[1.40 ; 1.50]$} & 1.43 & {$[0.77 ; 2.67]$} & 1.19 & ;1.28] \\
\hline & om. et chefs d'ent. & 1.34 & {$[1.05 ; 1.71]$} & 1.74 & {$[1.70 ; 1.79]$} & 1.23 & {$[0.72 ; 2.10]$} & 1.47 & {$[1.39 ; 1.55]$} \\
\hline & 4. Professions intermédiaires & 1.40 & {$[1.14 ; 1.70]$} & 1.47 & {$[1.44 ; 1.51]$} & 1.20 & {$[0.79 ; 1.82]$} & 1.46 & {$[1.40 ; 1.52]$} \\
\hline & 5. Employés & 2.20 & {$[1.77 ; 2.72]$} & 3.99 & {$[3.91 ; 4.08]$} & 1.39 & {$[0.95 ; 2.04]$} & 1.62 & {$[1.56 ; 1.69]$} \\
\hline & 6. Ouvriers & 2.10 & {$[1.75 ; 2.51]$} & 2.55 & {$[2.51 ; 2.60]$} & 1.80 & {$[1.19 ; 2.75]$} & 1.29 & {$[1.23 ; 1.35]$} \\
\hline & 8. In & 8.52 & {$[6.90 ; 10.53]$} & 13.60 & {$[13.33 ; 13.87]$} & 2.49 & {$[1.70 ; 3.63]$} & 4.68 & {$[4.50 ; 4.87]$} \\
\hline \multirow{6}{*}{$2000-2006$} & 1. Agriculteurs & 1.80 & {$[1.28 ; 2.54]$} & 2.26 & {$[2.18 ; 2.35]$} & 1.70 & {$[0.84 ; 3.45]$} & 1.52 & {$[1.40 ; 1.66]$} \\
\hline & 2. Artisans, com. et chefs d'ent. & 1.41 & {$[1.05 ; 1.88]$} & 1.76 & {$[1.71 ; 1.82]$} & 1.48 & {$[0.86 ; 2.56]$} & 1.53 & {$[1.44 ; 1.63]$} \\
\hline & 4. Professions intermédiaires & 1.42 & {$[1.13 ; 1.80]$} & 1.70 & {$[1.65 ; 1.74]$} & 1.01 & {$[0.68 ; 1.50]$} & 1.52 & {$[1.46 ; 1.59]$} \\
\hline & 5. Employés & 2.46 & {$[1.94 ; 3.12]$} & 3.87 & {$[3.77 ; 3.97]$} & 1.27 & {$[0.88 ; 1.82]$} & 1.91 & {$[1.84 ; 1.99]$} \\
\hline & 6. Ouvriers & 2.48 & {$[2.01 ; 3.06]$} & 3.85 & {$[3.76 ; 3.94]$} & 1.68 & {$[1.11 ; 2.52]$} & 2.44 & [2.33;2.55] \\
\hline & 8. Inactifs ${ }^{* *}$ & 8.29 & {$[6.56 ; 10.47]$} & 21.64 & [21.15;22.14] & 3.42 & [2.39; 4.89] & 7.93 & {$[7.63 ; 8.25]$} \\
\hline
\end{tabular}

${ }_{\star *}^{*}$ :Artisans, commerçants et chefs d'entreprise

: Inactifs, chômeurs n'ayant jamais travaillé et autres

Estimations de risques relatifs et d'intervalles de confiance issues de modèles log-linéaires de Poisson ajustés sur l'âge sans prise en compte de la surdispersion 
Tableau 5. Risque relatif de mortalité Ouvriers vs. Cadres et professions intellectuelles supérieures, selon l'approche et la période-par sexe et cause de décès

\begin{tabular}{|c|c|c|c|c|c|c|c|c|c|}
\hline \multirow[b]{2}{*}{ Période } & \multirow[b]{2}{*}{ Cause } & \multicolumn{4}{|c|}{ Homme } & \multicolumn{4}{|c|}{ Femme } \\
\hline & & \multicolumn{2}{|c|}{$\begin{array}{l}\text { Chaînée } \\
\text { (EDP) }\end{array}$} & \multicolumn{2}{|c|}{$\begin{array}{c}\text { Non chaînée } \\
\text { (Etat-civil) }\end{array}$} & \multicolumn{2}{|r|}{$\begin{array}{l}\text { Chaînée } \\
\text { (EDP) }\end{array}$} & \multicolumn{2}{|c|}{$\begin{array}{c}\text { Non chaînée } \\
\text { (Etat-civil) }\end{array}$} \\
\hline \multirow{8}{*}{$1983-1989$} & Toutes causes & 2.42 & {$[1.99 ; 2.94]$} & 2.85 & {$[2.79 ; 2.91]$} & 0.93 & {$[0.54 ; 1.62]$} & 1.18 & {$[1.12 ; 1.25]$} \\
\hline & Maladies infectieuses & - & & 0.75 & {$[0.66 ; 0.84]$} & - & & 1.22 & {$[0.73 ; 2.03]$} \\
\hline & Cancers & 1.49 & {$[1.05 ; 2.11]$} & 2.33 & {$[2.24 ; 2.42]$} & 0.44 & {$[0.18 ; 1.09]$} & 1.07 & {$[0.99 ; 1.16]$} \\
\hline & Maladies cardiovasculaires & 2.53 & {$[1.49 ; 4.30]$} & 2.54 & {$[2.41 ; 2.67]$} & - & & 1.71 & {$[1.43 ; 2.05]$} \\
\hline & Suicide & 2.26 & {$[1.26 ; 4.08]$} & 2.51 & {$[2.37 ; 2.67]$} & 0.54 & {$[0.12 ; 2.44]$} & 0.85 & {$[0.74 ; 0.99]$} \\
\hline & Autres causes externes & 2.54 & {$[1.48 ; 4.36]$} & 2.53 & {$[2.41 ; 2.65]$} & 1.02 & {$[0.32 ; 3.26]$} & 1.17 & {$[1.02 ; 1.33]$} \\
\hline & Alcool & 10.01 & {$[4.70 ; 21.34]$} & 11.13 & {$[10.21 ; 12.12]$} & - & & 4.65 & {$[3.42 ; 6.33]$} \\
\hline & Autres causes & 1.60 & {$[1.02 ; 2.50]$} & 2.46 & {$[2.32 ; 2.60]$} & 0.60 & {$[0.10 ; 3.58]$} & 1.07 & {$[0.93 ; 1.23]$} \\
\hline \multirow{8}{*}{$1991-1997$} & Toutes causes & 2.10 & {$[1.75 ; 2.51]$} & 2.55 & {$[2.51 ; 2.60]$} & 1.80 & {$[1.19 ; 2.75]$} & 1.29 & {$[1.23 ; 1.35]$} \\
\hline & Maladies infectieuses & 0.62 & {$[0.35 ; 1.10]$} & 0.70 & {$[0.66 ; 0.74]$} & - & & 0.90 & {$[0.69 ; 1.18]$} \\
\hline & Cancers & 1.85 & {$[1.33 ; 2.57]$} & 2.48 & {$[2.39 ; 2.57]$} & 1.08 & {$[0.61 ; 1.90]$} & 1.12 & {$[1.04 ; 1.20]$} \\
\hline & Maladies cardiovasculaires & 2.40 & {$[1.41 ; 4.06]$} & 2.58 & {$[2.45 ; 2.72]$} & - & & 2.12 & {$[1.78 ; 2.52]$} \\
\hline & Suicide & 3.36 & {$[1.84 ; 6.13]$} & 2.59 & {$[2.44 ; 2.74]$} & 3.30 & {$[0.72 ; 15.05]$} & 1.16 & {$[1.00 ; 1.36]$} \\
\hline & Autres causes externes & 1.65 & {$[1.06 ; 2.56]$} & 2.69 & {$[2.55 ; 2.83]$} & 1.62 & {$[0.51 ; 5.16]$} & 1.43 & {$[1.25 ; 1.64]$} \\
\hline & Alcool & 6.35 & {$[3.32 ; 12.12]$} & 8.72 & {$[8.06 ; 9.45]$} & - & & 3.45 & {$[2.68 ; 4.44]$} \\
\hline & Autres causes & 1.69 & {$[1.03 ; 2.79]$} & 2.33 & {$[2.21 ; 2.46]$} & 2.45 & {$[0.81 ; 7.40]$} & 1.14 & {$[1.00 ; 1.31]$} \\
\hline \multirow{8}{*}{$2000-2006$} & Toutes causes & 2.48 & {$[2.01 ; 3.06]$} & 3.85 & {$[3.76 ; 3.94]$} & 1.68 & {$[1.11 ; 2.52]$} & 2.44 & [2.33;2.55] \\
\hline & Maladies infectieuses & 1.65 & {$[0.36 ; 7.65]$} & 2.63 & {$[2.29 ; 3.03]$} & - & & 2.71 & {$[1.82 ; 4.05]$} \\
\hline & Cancers & 1.85 & {$[1.26 ; 2.70]$} & 3.42 & {$[3.28 ; 3.56]$} & 0.92 & {$[0.50 ; 1.70]$} & 2.08 & {$[1.96 ; 2.21]$} \\
\hline & Maladies cardiovasculaires & 3.49 & {$[1.87 ; 6.53]$} & 4.09 & {$[3.84 ; 4.35]$} & 1.07 & {$[0.37 ; 3.08]$} & 3.47 & {$[2.97 ; 4.05]$} \\
\hline & Suicide & 2.70 & {$[1.51 ; 4.82]$} & 3.92 & {$[3.68 ; 4.17]$} & 7.41 & {$[0.94 ; 58.47]$} & 2.01 & {$[1.75 ; 2.32]$} \\
\hline & Autres causes externes & 3.14 & {$[1.72 ; 5.71]$} & 3.85 & {$[3.62 ; 4.09]$} & 3.24 & {$[0.69 ; 15.26]$} & 3.07 & {$[2.64 ; 3.57]$} \\
\hline & Alcool & 4.07 & {$[2.12 ; 7.82]$} & 7.05 & {$[6.51 ; 7.63]$} & 5.57 & {$[0.69 ; 45.28]$} & 5.88 & {$[4.69 ; 7.38]$} \\
\hline & Autres causes & 1.79 & {$[1.13 ; 2.84]$} & 3.25 & {$[3.08 ; 3.43]$} & 2.60 & {$[0.95 ; 7.10]$} & 2.40 & {$[2.13 ; 2.70]$} \\
\hline
\end{tabular}

- : effectifs trop faibles, modèle non convergent

1 : sauf cancer des voies aérodigestives supérieures; 2 : causes externes sauf suicide; 3 : cancer des voix aérodigestives supérieures, psychoses alcoolique et alcoolisme, cirrhose alcoolique ou s.p. du foie

31 
Tableau 6. Risque relatif de mortalité par PCS vs. Cadres et professions intellectuelles supérieures par cause de décès selon l'approche - 2000-2006 - Hommes

\begin{tabular}{|c|c|c|c|c|c|c|c|c|}
\hline \multirow[b]{2}{*}{ Cause } & \multirow[b]{2}{*}{ PCS } & \multicolumn{3}{|c|}{ Homme } & \multicolumn{4}{|c|}{ Femme } \\
\hline & & $\begin{array}{l}\text { Chaînée } \\
\text { (EDP) }\end{array}$ & & $\begin{array}{l}\text { Non chaînée } \\
\text { (Etat-civil) }\end{array}$ & & $\begin{array}{c}\text { Chaînée } \\
\text { (EDP) }\end{array}$ & & $\begin{array}{l}\text { Non chaînée } \\
\text { (Etat-civil) }\end{array}$ \\
\hline \multirow{6}{*}{ Toutes causes } & 1. Agriculteurs exploitants & $1.80[1.28 ; 2.54]$ & 2.26 & {$[2.18 ; 2.35]$} & 1.70 & {$[0.84 ; 3.45]$} & 1.52 & {$[1.40 ; 1.66]$} \\
\hline & 2. Artisans, com. et chefs d'ent. & $1.41[1.05 ; 1.88]$ & 1.76 & {$[1.71 ; 1.82]$} & 1.48 & {$[0.86 ; 2.56]$} & 1.53 & {$[1.44 ; 1.63]$} \\
\hline & 4. Professions intermédiaires & $1.42[1.13 ; 1.80]$ & 1.70 & {$[1.65 ; 1.74]$} & 1.01 & {$[0.68 ; 1.50]$} & 1.52 & {$[1.46 ; 1.59]$} \\
\hline & 5. Employés & $2.46[1.94 ; 3.12]$ & 3.87 & {$[3.77 ; 3.97]$} & 1.27 & {$[0.88 ; 1.82]$} & 1.91 & {$[1.84 ; 1.99]$} \\
\hline & 6. Ouvriers & $2.48[2.01 ; 3.06]$ & 3.85 & {$[3.76 ; 3.94]$} & 1.68 & {$[1.11 ; 2.52]$} & 2.44 & {$[2.33 ; 2.55]$} \\
\hline & 8. Inactifs ${ }^{*}$ & $8.29[6.56 ; 10.47]$ & 21.64 & {$[21.15 ; 22.14]$} & 3.42 & {$[2.39 ; 4.89]$} & 7.93 & {$[7.63 ; 8.25]$} \\
\hline \multirow{6}{*}{ Cancers } & 1. Agriculteurs exploitants & $1.18[0.58 ; 2.39]$ & 1.47 & {$[1.36 ; 1.59]$} & 2.01 & {$[0.85 ; 4.79]$} & 1.24 & {$[1.09 ; 1.40]$} \\
\hline & 2. Artisans, com. et chefs d'ent. & $0.95[0.54 ; 1.67]$ & 1.40 & {$[1.32 ; 1.48]$} & 1.22 & {$[0.57 ; 2.62]$} & 1.34 & {$[1.22 ; 1.46]$} \\
\hline & 4. Professions intermédiaires & $1.13[0.73 ; 1.74]$ & 1.61 & {$[1.53 ; 1.68]$} & 1.02 & {$[0.60 ; 1.73]$} & 1.47 & {$[1.38 ; 1.55]$} \\
\hline & 5. Employés & $1.99[1.28 ; 3.10]$ & 3.49 & {$[3.34 ; 3.66]$} & 1.16 & {$[0.72 ; 1.89]$} & 1.74 & {$[1.64 ; 1.83]$} \\
\hline & 6. Ouvriers & $1.85[1.26 ; 2.70]$ & 3.42 & {$[3.28 ; 3.56]$} & 0.92 & {$[0.50 ; 1.70]$} & 2.08 & {$[1.96 ; 2.21]$} \\
\hline & 8. Inactifs ${ }^{\star \star}$ & $4.31[2.68 ; 6.91]$ & 12.28 & {$[11.78 ; 12.80]$} & 1.89 & {$[1.14 ; 3.12]$} & 4.96 & {$[4.70 ; 5.23]$} \\
\hline \multirow{6}{*}{$\begin{array}{l}\text { Maladies } \\
\text { cardiovasc. }\end{array}$} & 1. Agriculteurs exploitants & $2.13[0.79 ; 5.75]$ & 2.25 & {$[2.03 ; 2.50]$} & - & & 2.01 & {$[1.52 ; 2.65]$} \\
\hline & 2. Artisans, com. et chefs d'ent." & $1.99[0.89 ; 4.44]$ & 2.09 & {$[1.93 ; 2.27]$} & - & & 2.03 & {$[1.65 ; 2.50]$} \\
\hline & 4. Professions intermédiaires & $2.08[1.06 ; 4.11]$ & 1.88 & {$[1.75 ; 2.02]$} & - & & 1.57 & {$[1.35 ; 1.83]$} \\
\hline & 5. Employés & $2.45[1.17 ; 5.12]$ & 4.18 & {$[3.89 ; 4.48]$} & - & & 2.51 & {$[2.18 ; 2.90]$} \\
\hline & 6. Ouvriers & $3.49[1.87 ; 6.53]$ & 4.09 & {$[3.84 ; 4.35]$} & - & & 3.47 & {$[2.97 ; 4.05]$} \\
\hline & 8. Inactifs ${ }^{* *}$ & $9.53[4.70 ; 19.30]$ & 20.42 & {$[19.16 ; 21.76]$} & - & & 10.82 & {$[9.41 ; 12.45]$} \\
\hline \multirow{6}{*}{ Suicide } & 1. Agriculteurs exploitants & $3.10[1.36 ; 7.08]$ & 3.93 & {$[3.59 ; 4.30]$} & 6.45 & {$[0.40 ; 103.26]$} & 2.32 & {$[1.84 ; 2.93]$} \\
\hline & 2. Artisans, com. et chefs d'ent. & $2.25[1.08 ; 4.67]$ & 2.32 & {$[2.14 ; 2.52]$} & 7.67 & {$[0.80 ; 73.81]$} & 1.45 & {$[1.19 ; 1.77]$} \\
\hline & 4. Professions intermédiaires & $1.85[0.99 ; 3.47]$ & 1.73 & {$[1.62 ; 1.86]$} & 4.61 & {$[0.60 ; 35.46]$} & 1.57 & {$[1.38 ; 1.78]$} \\
\hline & 5. Employés & $3.03[1.60 ; 5.75]$ & 3.86 & {$[3.60 ; 4.14]$} & 4.01 & {$[0.54 ; 29.82]$} & 1.66 & {$[1.47 ; 1.88]$} \\
\hline & 6. Ouvriers & $2.70[1.51 ; 4.82]$ & 3.92 & {$[3.68 ; 4.17]$} & 7.41 & {$[0.94 ; 58.47]$} & 2.01 & {$[1.75 ; 2.32]$} \\
\hline & 8. Inactifs ${ }^{*}$ & $3.41[1.58 ; 7.40]$ & 19.03 & {$[17.84 ; 20.30]$} & 10.04 & {$[1.35 ; 74.52]$} & 7.07 & {$[6.27 ; 7.96]$} \\
\hline \multirow{6}{*}{$\begin{array}{l}\text { Autres causes } \\
\text { externes }\end{array}$} & 1. Agriculteurs exploitants & $1.37[0.44 ; 4.25]$ & 3.33 & {$[3.04 ; 3.66]$} & - & & 2.09 & {$[1.59 ; 2.76]$} \\
\hline & 2. Artisans, com. et chefs d'ent. & $1.40[0.59 ; 3.33]$ & 2.14 & {$[1.97 ; 2.32]$} & - & & 2.19 & {$[1.79 ; 2.67]$} \\
\hline & 4. Professions intermédiaires & $1.76[0.91 ; 3.40]$ & 1.62 & {$[1.51 ; 1.74]$} & - & & 1.58 & {$[1.37 ; 1.83]$} \\
\hline & 5. Employés & $2.39[1.21 ; 4.75]$ & 3.23 & {$[3.01 ; 3.47]$} & - & & 2.01 & {$[1.75 ; 2.30]$} \\
\hline & 6. Ouvriers & $3.14[1.72 ; 5.71]$ & 3.85 & {$[3.62 ; 4.09]$} & - & & 3.07 & {$[2.64 ; 3.57]$} \\
\hline & 8. Inactifs ${ }^{* *}$ & $6.26[3.11 ; 12.58]$ & 18.95 & {$[17.79 ; 20.19]$} & - & & 7.77 & {$[6.79 ; 8.89]$} \\
\hline \multirow{6}{*}{ Alcool } & 1. Agriculteurs exploitants & $3.86[1.61 ; 9.28]$ & 2.94 & {$[2.60 ; 3.32]$} & - & & 2.21 & {$[1.48 ; 3.29]$} \\
\hline & 2. Artisans, com. et chefs d'ent. & $1.81[0.77 ; 4.27]$ & 2.32 & {$[2.09 ; 2.56]$} & - & & 2.97 & {$[2.23 ; 3.95]$} \\
\hline & 4. Professions intermédiaires & $1.77[0.85 ; 3.67]$ & 2.22 & {$[2.03 ; 2.42]$} & - & & 1.98 & {$[1.57 ; 2.50]$} \\
\hline & 5. Employés & $4.17[2.04 ; 8.54]$ & 6.77 & {$[6.22 ; 7.37]$} & - & & 3.55 & {$[2.86 ; 4.42]$} \\
\hline & 6. Ouvriers & $4.07[2.12 ; 7.82]$ & 7.05 & {$[6.51 ; 7.63]$} & - & & 5.88 & {$[4.69 ; 7.38]$} \\
\hline & 8. Inactifs ${ }^{\star *}$ & $17.61[8.84 ; 35.06]$ & 48.02 & {$[44.39 ; 51.94]$} & - & & 28.63 & {$[23.16 ; 35.40]$} \\
\hline \multirow{6}{*}{ Autres causes } & 1. Agriculteurs exploitants & $1.08[0.44 ; 2.67]$ & 1.71 & {$[1.55 ; 1.89]$} & 1.23 & {$[0.14 ; 10.55]$} & 1.43 & {$[1.13 ; 1.81]$} \\
\hline & 2. Artisans, com. et chefs d'ent. & $1.03[0.52 ; 2.04]$ & 1.37 & {$[1.27 ; 1.48]$} & 1.00 & {$[0.19 ; 5.14]$} & 1.30 & {$[1.09 ; 1.55]$} \\
\hline & 4. Professions intermédiaires & $1.01[0.59 ; 1.72]$ & 1.53 & {$[1.44 ; 1.63]$} & 0.69 & {$[0.23 ; 2.06]$} & 1.51 & {$[1.35 ; 1.69]$} \\
\hline & 5. Employés & $1.99[1.16 ; 3.39]$ & 3.62 & {$[3.41 ; 3.85]$} & 0.95 & {$[0.37 ; 2.49]$} & 1.96 & {$[1.77 ; 2.18]$} \\
\hline & 6. Ouvriers & $1.79[1.13 ; 2.84]$ & 3.25 & {$[3.08 ; 3.43]$} & 2.60 & {$[0.95 ; 7.10]$} & 2.40 & {$[2.13 ; 2.70]$} \\
\hline & 8. Inactifs ${ }^{* *}$ & $12.31[7.63 ; 19.87]$ & 30.83 & {$[29.25 ; 32.49]$} & 6.36 & {$[2.57 ; 15.77]$} & 13.13 & {$[11.87 ; 14.52]$} \\
\hline
\end{tabular}

1 : sauf cancer des voies aérodigestives supérieures; 2 : causes externes sauf suicide; 3 : cancer des voix aérodigestives supérieures, psychoses alcoolique et alcoolisme, cirrhose alcoolique ou s.p. du foie ; Inactifs, chômeurs n'ayant jamais travaillé et autres

:Artisans, commerçants et chefs d'entreprise

: Inactifs, chômeurs n'ayant jamais travaillé et autres 